\title{
THE REVE'S PUZZLE WITH AN EVILDOER DISC
}

\author{
A.A.K. MAJUMDAR \\ Ritsumeikan Asia-Pacific University, Beppu-shi 874-8577, Japan
}

\begin{abstract}
This paper considers a variant of the Reve's puzzle with $n(\geq 1)$ discs and an evildoer, which can be placed directly on top of a smaller disc any number of times. Denoting by $E(n)$ the minimum number of moves required to solve the new variant, we give a scheme to find the optimality equation satisfied by $E(n)$. We then find an explicit form of $E(n)$.
\end{abstract}

Keywords: Reve's puzzle, divine rule, evildoer

\section{INTRODUCTION}

The classical Tower of Hanoi problem, introduced by the famous French number theorist Lucas (1883), in general form, is as follows : Given are $n(\geq 1)$ discs of different sizes, labeled from the smallest to the largest as $D_{1}, D_{2}, \ldots, D_{n}$, and three pegs, $S, P$ and $D$. At the start of the game, the discs rest on the source $p e g, S$, in a tower in increasing order, from top to bottom. The objective is to shift the tower (from the peg $S$ ) to the destination peg, $D$, in minimum number of moves, where each move can shift only the topmost disc from one peg to another, under the "divine rule" that no disc can ever be placed on a smaller one at any stage of the transfer process. It is well-known that the total number of moves required to solve the classical Tower of Hanoi problem with $n(\geq 1)$ discs is $2^{n}-1$.

Over the past decades, the classical Tower of Hanoi problem has seen many variants, some of which have been reviewed by Majumdar (2012, 2013) and Hinz, Klavzar and Petr (2018). Chen, Tian and Wang (2007) have introduced a new variant of the Tower of Hanoi problem which allows $r(\geq 1)$ violations of the "divine rule". In the new variant, the problem is to shift the tower of $n$ discs from the peg $S$ to the peg $D$ in minimum number of moves, where for (at most) $r$ moves, some disc may be placed directly on top of a smaller one.

Denoting by $S_{3}(n, r)$ the minimum number of moves required to solve the new variant, $S_{3}(n, r)$ is given in the following lemma, due to Chen, Tian and Wang (2007).

Theorem 1.1 :For any $n \geq 1, r \geq 1$,

$$
S_{3}(n, r)= \begin{cases}2 n-1, & \text { if } 1 \leq n \leq r+2 \\ 4 n-2 r-5, & \text { if } r+2 \leq n \leq 2 r+3 \\ 2^{n-2 r}+6 r-1, & \text { if } n \geq 2 r+3\end{cases}
$$

Chen, Tian and Wang (2007) have posed a new variant of the classical Tower of Hanoi problem with $n(\geq 1)$ discs, $r(\geq 1)$ of which are evildoers, where each evildoer can be placed on top of a smaller one any number of times. Thus, in the new variant, the problem is to shift the tower of $n$ discs from the peg $S$ to the peg $D$ in minimum number of moves, where any of the $r$ evildoers may be placed directly on top of a smaller one any number of times (thereby violating the "divine rule"). The problem was taken up by Majumdar and Islam (2019). Denoting by $E_{3}(n)$ the minimum number of moves required to solve 
the classical Tower of Hanoi problem with one evildoer disc, the explicit form is given as follows.

Proposition 1.1 : For $n \geq 8$,

$$
E_{3}(n)= \begin{cases}2^{n-3}+2^{\frac{n}{2}}+9, & \text { if } n \text { is even } \\ 2^{n-3}+3.2^{\frac{n-3}{2}}+9, & \text { if } n \text { is odd }\end{cases}
$$

with

$$
E_{3}(n)=S_{3}(n, 1) \text { for } 1 \leq n \leq 7 .
$$

Moreover, the disc $D_{n-2}$ is the (unique) evildoer.

Corollary 1.1 : For $n \geq 8$,

$$
E_{3}(n+1)-E_{3}(n)=2^{n-3}+2^{m-1},
$$

where

$$
n=2 m+j,
$$

for some integers $m$ and $j, j \in\{0,1\}$.

Corollary 1.1 above has the consequence below.

Corollary 1.2 :For $n \geq 1, E_{3}(n)$ is strictly convex in $n$ in the sense that

$$
E_{3}(n+2)-E_{3}(n+1)>E_{3}(n+1)-E(n) .
$$

The Reve's puzzle, due to Dudeney (1958), is the Tower of Hanoi problem with four pegs. The initial state of the Reve's puzzle is shown below, where the objective is to transfer the tower of $n$ discs (from the source peg $S$ ) to the destination peg $D$, in minimum number of moves, where each move transfers only the topmost disc from one peg to another under the "divine rule" that no disc is ever placed on top of a smaller one.

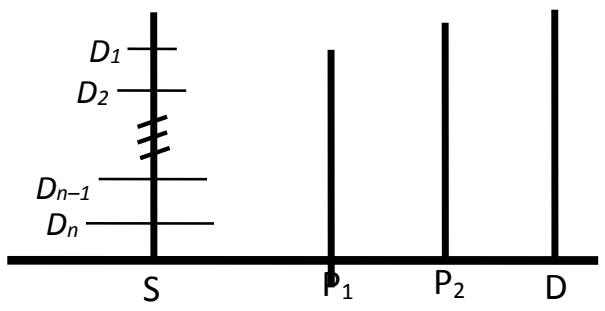

This paper considers the Reve's puzzle with one evildoer disc, which can be placed on top of a smaller disc any number of times. Denoting by $E(n)$ the minimum number of moves required to solve this variant of the Reve's puzzle, we find an expression of $E(n)$. This is done in Proposition 3.3 in the third Section. The next Section gives some background materials. Some remarks are made in the final section.

\section{BACKGROUND MATERIAL}

This section gives some background materials that would be required later.

Let $M(n)$ denote the minimum number of moves required to solve the Reve's puzzle with $n(\geq 1)$ discs. Then, $M(n)$ satisfies the dynamic programming equation below for $n(\geq 4)$ (see, for example, Chapter 3 in Majumdar (2012) :

$$
M(n)=\min _{1 \leq k \leq n-1}\left\{2 M(k)+2^{n-k}-1\right\},(1.1 \mathrm{a})
$$

with

$$
M(0)=0 ; M(n)=2 n-1 \text { for } 1 \leq n \leq 3 .(1.1 \mathrm{~b})
$$

The optimal value function $M(n)$ satisfies the local-value relationships given in Lemma 2.1 below. The solution of the dynamic programming equation (1.1) is given in Theorem 2.1. For proofs of these results, we refer the reader to Majumdar (2012).

Lemma 2.1 : Exactly one of the following two relationships hold :

(a) $M(n+2)-M(n+1)=M(n+1)-M(n)$,

(b) $M(n+2)-M(n+1)=2[M(n+1)-M(n)]$.

Theorem 2.1 : The solution of (1.1) is as follows. 
(a) For $s \geq 1, M\left(\frac{s(s+1)}{2}\right)$ is attained at the unique point $k=\frac{s(s-1)}{2}$, with

$$
M\left(\frac{s(s+1)}{2}\right)=(s-1) 2^{s}+1 .
$$

(b) Let, for some $\mathrm{s} \in\{1,2, \ldots\}$,

$$
\frac{s(s+1)}{2}<n<\frac{(s+1)(s+2)}{2} .
$$

Then, $M(n)$ is attained at the two points $k=n-s-1, n-s$, with

$$
M(n)=\left\{n-\frac{s(s-1)}{2}-1\right\} 2^{\mathrm{s}}+1 .
$$

Corollary 2.1 : For $3 \leq n \leq 6, M(n)=4 n-7$.

Proof : From Theorem 2.1, we see that

$$
M(3)=5, M(4)=9, M(5)=13, M(6)=17 \text {. }
$$

This establishes the desired result.

Over the past decades, the Reve's puzzle has seen several generalizations, some of which have been reviewed by Majumdar (2013). Recently, Majumdar (2019) has considered a variant of the Reve's puzzle which allows one violation of the "divine rule". Denoting by $S(n)$ the minimum number of moves required to solve the Reve's puzzle with single relaxation of the "divine rule", an explicit form of $S(n)$ is given below.

Theorem 2.2 : For $n \geq 1$,

$$
S(n)= \begin{cases}2 n-1, & \text { if } 1 \leq n \leq 4 \\ M(n-1)+2, & \text { if } 4 \leq n \leq 7 \\ M(n-2)+6, & \text { if } n \geq 7\end{cases}
$$

In view of Corollary 2.1, Theorem 2.2 has the following equivalent form.

Theorem 2.3 : For $n \geq 1$,

$$
S(n)= \begin{cases}2 n-1, & \text { if } 1 \leq n \leq 4 \\ 4 n-9, & \text { if } 4 \leq n \leq 7 \\ M(n-2)+6, & \text { if } n \geq 7\end{cases}
$$

\section{THE PROBLEM AND THE SOLUTION}

The problem that we consider is as follows: There are four pegs, $S, P_{1}, P_{2}$ and $D$, and $n(\geq 1)$ discs of varying sizes. Initially, the discs rest on the source peg $S$ in a tower, in increasing order from top to bottom. Of the $n$ discs, one disc is evildoer, which can be placed directly on top of a smaller one any number of times. The problem is to shift the tower from the peg $S$ to the destination peg $D$, in minimum number of moves, such that each move shifts only the topmost disc from one peg to another.

From Theorem 2.3, we see that

$$
S(n)=M(n-2)+6, n \geq 8,
$$

where the optimal scheme is as follows :

Step 1 : Move the topmost $K$ discs (from the peg $S$ ) to one of the two auxiliary pegs, say, $P_{1}$, in $M(K)$ moves,

Step 2 : Shift the remaining $n-K$ discs, now resting on the peg $S$, to the peg $D$, using the three pegs available, in $S_{3}(n-K, 1)$ moves,

Step 3 : Transfer the tower of $K$ discs on the peg $P_{1}$ to the peg $D$ (again in $M(K)$ moves) to complete the tower on the destination peg $D$. Therefore,

$$
S(n)=2 M(K)+S_{3}(n-K, 1),
$$

where the integer $K$ is such that

$$
M(n-2)=2 M(K)+2^{n-K-2}-1 \text {. }
$$

Recall that, if $n-2$ is not a triangular number, then $M(n-2)$ is attained at two values of $k$, say, at $k=K, K+1$. In such a case, 
$S(n)=2 M(K+1)+S_{3}(n-K-1,1)$.

Therefore, if $n-2$ is not a triangular number, then from (3.1) and (3.2), we get

$$
\begin{array}{r}
2[M(K+1)-M(K)]=S_{3}(n-K, 1) \\
-S_{3}(n-K-1,1)=2^{n-K-3} .
\end{array}
$$

Let the minimum number of moves required to solve the above problem be $E(n)$. Clearly,

$E(n)=S(n)$ for all $1 \leq n \leq 7$.

When $8 \leq n \leq 17$, so that $6 \leq n-2 \leq 15$, it can easily be verified, using Theorem 2.1, that

$$
n-K=n-k_{\min }(n-2) \leq 7 .
$$

Thus,

$$
E_{3}(n-K)=S_{3}(n-K, 1) .
$$

Therefore, by (3.1),

$$
E(n)=S(n), \text { if } 8 \leq n \leq 17 .
$$

The optimality equation satisfied by $E(n)$ is given below.

Proposition 3.1 : For $n \geq 18$,

$$
E(n)=\min _{1 \leq k \leq n-8}\left\{2 M(k)+E_{3}(n-k)\right\} .
$$

Proof : To find $E(n)$ for $n \geq 18$, we follow the scheme below :

Step 1 : Move the topmost $k(\geq 1)$ smallest discs (from the peg $S$ ) to some auxiliary peg, say, $P_{1}$, in $M(k)$ number of moves,

Step 2 : Transfer the remaining $n-k$ discs (from the peg $S$ ) to the peg $D$, using the evildoer, in $E_{3}(n-k)$ number of moves,

Step 3 : Shift the $k$ discs (from the peg $P_{l}$ ) to the peg $D$, in $M(k)$ number of moves, to complete the tower on the peg $D$.

The total number of moves involved in the above three steps is
$2 M(k)+E_{3}(n-k)$

where $k(1 \leq k \leq n-8)$ is to be determined such that the above expression is minimum. This establishes the proposition.

In Proposition 3.1 above, we require that $k \leq n-8$ to guarantee that

$$
E_{3}(n-k)<S_{3}(n-k, 1) .
$$

For any $n \geq 1$ fixed, let the function $F(n, k)$ be defined as follows :

$$
F(n, k)=2 M(k)+E_{3}(n-k), 0 \leq k \leq n .
$$

Then, we have the following result which states that (for fixed $n), F(n, k)$ is strictly convex in $k$, in the sense of the inequality stated therein.

Lemma 3.1 : For any $n \geq 2$ fixed,

$$
F(n, k+2)-F(n, k+1)>F(n, k+1)-F(n, k) .
$$

Proof : By (3.4),

$$
\begin{aligned}
& F(n, k+2)-F(n, k+1) \\
& =2[M(k+2)-M(k+1)]-
\end{aligned}
$$

$\left[E_{3}(n-k-1)-E_{3}(n-k-2)\right]$.

Using Lemma 2.1 and Corollary 1.2, we get the following chain of inequalities:

$$
\begin{aligned}
& F(n, k+2)-F(n, k+1) \\
= & 2[M(k+2)-M(k+1)]- \\
& {\left[E_{3}(n-k-1)-E_{3}(n-k-2)\right] } \\
> & 2[M(k+1)-M(k)]- \\
& {\left[E_{3}(n-k)-E_{3}(n-k-1)\right] . } \\
= & F(n, k+1)-F(n, k) .
\end{aligned}
$$

Thus, the lemma is established.

An explicit form of $E(n)$ is given in Proposition 3.2 below. 
Proposition 3.2 : If $1 \leq n \leq 17$,

$$
E(n)=S(n),
$$

and for $n \geq 18$,

$$
E(n)=2 M(K)+E_{3}(n-K),
$$

where

$$
K=k_{\min }(n-2),
$$

$\left(k_{\min }(n-2)\right.$ being the minimum of the two values of $k$ at which $M(n-2)$ is attained), and $D_{n-2}$ is the evildoer.

Proof: When $18 \leq n \leq 23$, by Theorem $2.1, n-K=8$. Then, we have

$$
\begin{aligned}
& 2 M(K)+E_{3}(n-K) \\
& <2 M(K)+S_{3}(n-K, 1) \\
& \leq 2 M(K+1)+S_{3}(n-K-1,1) \\
& =2 M(K+1)+E_{3}(n-K-1)
\end{aligned}
$$

where the last equality holds by virtue of Proposition 1.1 (since $n-K-1=7$ ), and in (3.5), the strict equality sign holds by (3.3) if $M(n-2)$ is not a triangular number, and strict inequality sign otherwise. Also,

$$
\begin{aligned}
& 2 M(K)+E_{3}(n-K) \\
& <2 M(K)+S_{3}(n-K, 1)=S(n) .
\end{aligned}
$$

By Proposition 1.1, the disc $D_{n-2}$ is the evildoer. When $n \geq 24, n-K \geq 9$. In this case, we show that

$$
\begin{aligned}
& 2 M(K)+E_{3}(n-K) \\
& <2 M(K+1)+E_{3}(n-K-1)
\end{aligned}
$$

that is,

$$
\begin{aligned}
& 2[M(K+1)-M(K)] \\
& >E_{3}(n-K, 1)-E_{3}(n-K-1) .
\end{aligned}
$$

Now, if $n-K$ is even, say, $n-K=2 m$ for some integer $m>2$, then by virtue of Proposition 1.1,

$$
E_{3}(n-K)-E_{3}(n-K-1)=2^{2 m-4}+2^{m-2},
$$

and by (3.3),

$$
2[M(K+1)-M(K)]=2^{2 m-3} .
$$

On the other hand, if $n-K=2 m+1$ for some integer $m>2$, then

$$
E_{3}(n-K)-E_{3}(n-K-1)=2^{2 m-3}+2^{m-1},
$$

and

$$
2[M(K+1)-M(K)]=2^{2 m-2} .
$$

Thus, in either case, the inequality (3.6) is satisfied.

Next, we show that

$$
\begin{aligned}
& 2 M(K)+E_{3}(n-K) \\
& <2 M(K-1)+E_{3}(n-K+1) .
\end{aligned}
$$

The proof is by contradiction. So, let

$$
\begin{aligned}
& 2 M(K)+E_{3}(n-K) \\
& \geq 2 M(K-1)+E_{3}(n-K+1) .
\end{aligned}
$$

Then,

$$
\begin{aligned}
& 2[M(K)-M(K-1)] \\
\geq & E_{3}(n-K+1)-E_{3}(n-K)>2^{n-K-3},
\end{aligned}
$$

where the last inequality follows by virtue of Corollary 1.1 .

Now, since

$$
\begin{aligned}
S(n) & \equiv 2 M(K)+S_{3}(n-K, 1) \\
& <2 M(K-1)+S_{3}(n-K+1,1),
\end{aligned}
$$

we get the following inequality :

$$
\begin{gathered}
2[M(K)-M(K-1)]<S_{3}(n-K+1,1)- \\
S_{3}(n-K, 1)=2^{n-K-2} .
\end{gathered}
$$

Since (by Lemma 2.2 in Majumdar (2012)),

$2[M(K)-M(K-1)]=2^{s}$ for some integer $s \geq 1$, 
the inequalities in (1) and (2) together give the following chain of inequalities :

$$
2^{n-K-3}<2^{s}<2^{n-K-2},
$$

and we are led to a contradiction. This establishes the inequality (3.7).

It now follows from Lemma 3.1 that $2 M(k)+E_{3}(n-k)$ is minimized at the unique point $k=K$.

\section{CONCLUDING REMARKS}

This paper deals with a variant of the Reve's puzzle, when there is an evildoer disc which can be placed on a smaller disc any number of times. Denoting by $E(n)$ the minimum number of moves required to solve this problem, $E(n)$ is given explicitly in Proposition 3.2. It is interesting to note that $E(n)$ has a simple form, and further that $E(n)$ is given in terms of the optimal value function satisfied for the Reve's puzzle. Then, using Theorem 2.1, E(n) may be calculated. For example, for $n=18$, we get from Theorem 2.1 that $k_{\min }(16)=10$. Therefore, by Proposition 3.2,

$$
E(18)=2 M(10)+E_{3}(8) .
$$

Now, by Theorem 2.1, $M(10)=49$, and by Proposition 1.1, $E_{3}(8)=57$. Therefore,

$$
E(18)=135 \text {. }
$$

This number may be compared with $M(18)=225$, which is the number of moves required to solve the Reve's puzzle with 18 discs.

\section{REFERENCES}

Chen, X., Tian, B. and Wang, L. 2007. Santa Claus' Towers of Hanoi. Graphs and Combinatorics, 23 (Supplement): 15-167.

Claus, N. (= Lucas, E.) 1883. La Tour d Hanoi. Jeu de Calcul. Sci. Nat., 1(8): 127-128.

Dudeney, H. E. 1958. The Canterbury Puzzles. Dover, $4^{\text {th }}$ Edition.

Hinz, A.M., Klavzar, S. and Petre, C. 2018. The Tower of Hanoi-Myths and Maths, Springer, Basel.

Majumdar, A.A.K., 2012. The Classical Tower of Hanoi Problem and Its Generalizations, Vol.1: Multi-Peg Generalizations. Lambert Academic Publishing, U.S.A.

Majumdar, A.A.K., 2013. The Classical Tower of Hanoi Problem and Its Generalizations, Vol.2: Other Generalizations. Lambert Academic Publishing, U.S.A.

Majumdar, A.A.K. and Islam, S.M.S. 2019. The Tower of Brahma with One Evildoer: The Life-Time of the World. Journal of Science and Technology, 17: 111-118.

Majumdar, A.A.K., 2019. The Reve's Puzzle with Single Relaxation. J. Bangladesh Academy of Sciences, 43(2): 211-212 (Short Communication).

(Received 12 December 2019; revised manuscript on 15 May 2020) 